\title{
The role of topical vitamin A in promoting healing in surface refractive procedures: a prospective randomized controlled study
}

This article was published in the following Dove Press journal:

Clinical Ophthalmology

23 September 2013

Number of times this article has been viewed

\author{
Elias Chelala' \\ Ali Dirani' \\ Ali Fadlallah' \\ Sharbel Fahd ${ }^{2}$ \\ 'Saint Joseph University, Faculty \\ of Medicine, Beirut, Lebanon; \\ ${ }^{2}$ Ophthalmic Consultant of Beirut, \\ Chairman Ophthalmology, Lebanese \\ American University, Beirut, Lebanon
}

Correspondence: Ali Fadlallah Saint Joseph University, Faculty of Medicine, Rue de Damas, BP I I-5076, Riad El Solh, Beirut II 072180 , Lebanon Tel +96171388893

Email fadlallah2000@hotmail.com
Aim: To evaluate the effect of topical vitamin A supplementation on corneal re-epithelialization time, postoperative pain, visual acuity, and haze following photorefractive keratectomy (PRK).

Patients and methods: This prospective study included 32 patients. For each patient, one eye was randomized to the vitamin A group and the fellow eye to the non-vitamin A group (control group). Eyes in the vitamin A group received perioperative topical vitamin A (retinol palmitate, $250 \mathrm{IU} / \mathrm{g}$ VitAPOS eye ointment [AFT Pharmaceuticals Pty, Ltd, Sydney, NSW, Australia]) in addition to the classic treatment for PRK. Clinical outcomes were evaluated up to 3 months after PRK.

Results: There was no difference in the mean time to complete healing between the vitamin A group and the control group (3.36 \pm 0.6 days in the control group; $3.42 \pm 0.7$ days in the vitamin A group; $P=0.854$ ). Mean postoperative pain at the 48 -hour visit was $4.35 \pm 1.42$ over 10 in the control group, and $4.42 \pm 1.37$ over 10 in the vitamin A group, with no difference between the two groups $(P=0.589)$. Subepithelial haze evaluated at 3 months postoperatively did not differ between the two groups $(P=0.960)$. Also, visual and refractive outcomes were not different between the two groups 3 months postoperatively.

Conclusion: Topical vitamin A supplementation did not affect re-epithelialization time, postoperative pain, corneal haze formation, or visual outcomes after PRK.

Keywords: photorefractive keratectomy, topical vitamin A, corneal re-epithelialization

\section{Introduction}

Surface ablation has been proven to be an effective means of correcting error of refraction; it is less invasive than laser in situ keratomileusis and does not structurally weaken the cornea. ${ }^{1}$ However, it is associated with significant postoperative pain, slower visual recovery, and corneal haze. Several modifications of the conventional photorefractive keratectomy (PRK) procedure have been introduced to reduce the side effects of surface ablation. Most of these modification focused on the ways of removing the corneal epithelium (for example, Amoils brush [Innovative Excimer Solutions, Toronto, OT, Canada], mechanical abrasion, alcohol use, excimer laser), but very few studies focused on promoting epithelial healing after the surgery. ${ }^{2}$

Epithelial healing promoting factors such as vitamin A play an essential role in epithelial growth and limbal stem cell differentiation, and therefore promote corneal wound healing. ${ }^{3}$ The lack of vitamin $\mathrm{A}$ in corneal tissues can result in abnormal differentiation of epithelial cells, resulting in keratinization, ulceration, epithelial squamous metaplasia, and a deficiency of conjunctival goblet cells. ${ }^{4-6}$ The recognition of xerophthalmia and keratomalacia, related to vitamin A deficiency, as an important 
public health problem in many developing countries triggered many studies on the use of retinoids for ocular surface diseases. ${ }^{6}$ For this reason, topical retinoids have been tested as pharmacological agents for the treatment of ocular surface diseases involving wound healing, squamous metaplasia, or dry eye; the results of the studies were encouraging. ${ }^{7-9}$

Vitamin A used topically and orally has been reported to speed epithelial healing, but there is no study in the literature to date that has examined the role of topical vitamin A in promoting epithelial healing after refractive surgery. There is only one clinical trial in the literature of oral high-dose vitamin A and vitamin E supplementation after PRK, and the study showed faster epithelial healing when oral vitamin A and vitamin $\mathrm{E}$ were used. ${ }^{10}$ The present study compares epithelial healing, postoperative pain, and the incidence of haze on surface ablated corneas in refractive surgery when vitamin A (retinol palmitate) is used topically.

\section{Patients and methods}

This prospective, comparative, non-blinded study included 32 consecutive myopic patients (spherical equivalence less than -1.00 diopters [D]) operated using PRK at the Laser Vision Center, Beirut, Lebanon, between July 2012 and December 2012. In addition to the routinely prescribed regimen post-PRK surgery, randomization was done to add topical vitamin A to the treatment between the first and the fellow eye; 32 eyes were treated by the classic regimen post-PRK surgery (non-vitamin A/control group: 16 right eyes and 16 left eyes) and 32 fellow eyes (vitamin A group: 16 right eyes and 16 left eyes) were given topical vitamin A preoperatively and postPRK surgery (in addition to the classic regimen). All surgeries were done by the same surgeon (EC).

Preoperative examinations included a full eye examination with uncorrected visual acuity (UCVA) and best spectacle-corrected visual acuity (BCVA), manifest and cycloplegic refraction, slit-lamp examination, pachymetry, applanation tonometry, Scheimpflug analysis video keratometry readings (Galilei; Ziemer Ophthalmic Systems AG, Port, Switzerland), ocular aberrations (Ocular Wavefront Analyzer; Schwind Eye-Tech Solutions, GmbH, Kleinostheim, Germany), and a fundus examination. Tear function was assessed using a Schirmer's test without corneal anesthesia, and the fluorescein tear breakup time. We also studied the corneal tear film and ocular surface regularity with fluorescein staining.

The physician explained the procedure to the patients and answered all their questions. All patients signed informed consent about the procedure before surgery, and agreed to be included in this study. The review board committee of the Laser Vision Center, Beirut, Lebanon, approved the study.

The exclusion criteria were: patients with unstable errors of refraction, those with spherical equivalent errors $>6.0 \mathrm{D}$, those with anterior segment pathology or with any evidence of lid disease, patients with keratoconus or any irregularity in the topography, those with a past history of keratitis, and those who had previous intraocular or corneal surgeries. Soft contact lenses were discontinued for a minimum of 3 days, and hard lenses for a minimum of 3 weeks before examination and treatment.

\section{Surgical procedure}

Patients were given $5 \mathrm{mg}$ of diazepam (Valium ${ }^{\circledR}$, Roche, Italy) orally 30 to 60 minutes before the start of the procedure. One drop of proparacaine (Novocaine ${ }^{\circledR}$, Hospira, Inc., Lake Forest, IL, USA) and one drop of ofloxacin (Oflox ${ }^{\circledR}$; Allergan Pharmaceuticals, Irvine, CA, USA) were instilled in the eye on three occasions at 5-minute intervals. The eyes were then scrubbed and draped, and a closed-loop lid speculum equipped with suction was placed between the lids of the eye to be treated. The other eye was occluded. The laser used was the VISX S4 Custom Vue Laser (Abbott Medical Optics, Santa Ana, CA, USA). The optical zone was between $6 \mathrm{~mm}$ and $7 \mathrm{~mm}$ in both series, and the transition zone (varying from $0.36 \mathrm{~mm}$ to $2.24 \mathrm{~mm}$ ) was calculated according to the nomogram of the ORK-CAM (optimized refractive keratectomy - custom ablation manager) based on age, keratometric readings, and the error of refraction.

PRK was performed by placing an $8 \mathrm{~mm}$ or $9 \mathrm{~mm}$ well on the cornea. Twenty percent of ethyl alcohol was used for 25 seconds to 30 seconds to loosen the epithelium, which was then scrapped off using a WEDK-CEL ${ }^{\circledR}$ sponge (Beaver-Visitec, Waltham, MA, USA). The cornea was rinsed with balanced salt solution, and a dry MEROCEL sponge (Beaver-Visitec) was used to peel the epithelium from the eye. The eye was focused and centered on the optical axis and the treatment was delivered. Intraoperative and postoperative pain was assessed at the end of surgery, and was monitored daily until full epithelial healing was noted using the Visual Assessment Pain Score (VAPS), which measures subjective pain intensity on a scale from 0 (no pain) to 10 (worst pain). This scale was validated for the assessment of pain scores. ${ }^{11}$

Haze was graded using the system described by Fantes et $\mathrm{al}^{12}(0=$ no haze $+0.5=$ trace haze on oblique illumination; $+1=$ corneal cloudiness not interfering with the visibility of fine iris details; $+2=$ mild effacement of fine iris details; 
+3 and $+4=$ details of the lens and iris not discernible). The evaluation was done by the same ophthalmologist, and values at 3 months were considered for comparison between the two categories.

\section{Postoperative care and follow-up}

After laser ablation, a high-oxygen-content $(50 \%)$ soft contact lens was placed on the cornea (Acuvue ${ }^{\circledR}$ Oasys $^{\circledR}$; Johnson \& Johnson, Jacksonville, FL, USA), followed by one drop each of a topical antibiotic (ofloxacin, Oflox ${ }^{\circledR}$; Allergan Inc, Irvine, CA, USA), a topical steroid (PRED FORTE ${ }^{\circledR}$, Allergan Inc), and a topical nonsteroidal anti-inflammatory (Indocollyre; Bausch \& Lomb, Hong Kong). Patients were given prednisone drops to use four times per day, gatifloxacin drops to use four times per day, nonsteroidal anti-inflammatory drops to use three times per day, and preservative-free artificial tears to use every hour.

The vitamin A group treatment consisted of starting retinol palmitate to the eye at concentrations of $250 \mathrm{IU} / \mathrm{g}$ of a petrolatum-based ophthalmic ointment vehicle (VitA-POS ${ }^{\circledR}$ ointment; Urspharm GmbH \& Co, KG, Saarbrücken, Germany) four times per day for 4 days prior to the day of surgery and continued for up to 2 weeks postoperatively. The non-vitamin A group (control group) did not receive any. Epithelial healing was assessed on the second day postoperatively (at 48 hours), then daily using the slit-lamp and fluorescein staining, until complete epithelial healing occurred. Epithelial defect and healing were recorded as a sketch on the patient's chart. An epithelial-healing score was devised, rating the healing from 0 to 10 over time, depending on the defect remaining (10 is equivalent to complete healing $-100 \%$ of the initial total epithelial defect [remaining at the end of surgery] has healed, and 0 is equal to total closure of the defect). The daily subjective assessment of pain reported by the patient using the VAPS was also taken.

Once the epithelium had completely healed, the therapeutic contact lens was removed from the cornea, the nonsteroidal drops were stopped, the antibiotic drops and topical vitamin A were kept for an additional 4 days, while the steroid drops were tapered progressively over the next 4 weeks. On each follow-up visit (on day 2, daily until the epithelium healed; at 1 week; at 1 month; and at 3 months), UCVA, BCVA, and corneal clarity were assessed.

\section{Statistical analysis}

Statistical analysis was performed using the Statistical Package for the Social Sciences version 13.0 (SPSS version 13.0; SPSS Inc., Chicago, IL, USA). The Student's $t$-test for unpaired data was used to compare all parameters between the two groups. A chi-square test was used to compare the association between categorical variables. $P$-values $<0.05$ were considered statistically significant.

\section{Results}

\section{Preoperative patient characteristics}

The average preoperative UCVA was $0.29 \pm 0.14$ in the control group and $0.31 \pm 0.1$ in the vitamin A group $(P=0.543)$. The average preoperative BCVA was $0.018 \pm 0.003$ in the control group and $0.02 \pm 0.002$ in the vitamin A group $(P=0.267)$. Preoperative intraocular pressure, central corneal thickness, and topography-simulated keratometric values were $14.8 \pm 1.2 \mathrm{mmHg}, 522 \pm 32.6 \mu \mathrm{m}$, and 42.51/44.23 D, respectively, in the control group, and $13.68 \pm 3.2 \mathrm{mmHg}$, $519 \pm 28 \mu \mathrm{m}$, and $42.16 / 44.41 \mathrm{D}$, respectively, in the vitamin A group $(P=0.672, P=0.749, P=0.860$, and $P=0.865$, respectively). Mean preoperative Schirmer examinations without anesthesia were $15.72 \pm 2.6 \mathrm{~mm} / 5$ minutes in the control group, and $16.89 \pm 1.8 \mathrm{~mm} / 5$ minutes in the vitamin A group $(P=0.402)$.

The average spherical error was $-3.22 \pm 1.4 \mathrm{D}$ in the control group, and $-3.51 \pm 1.2 \mathrm{D}$ in the vitamin A group $(P=0.429)$. The average cylinder in the control group was $0.97 \pm 0.7 \mathrm{D}$, whereas in the vitamin A group it was $0.75 \pm 0.6 \mathrm{D}(P=0.910)$. Mean treatment optical zone was $6.85 \mathrm{~mm}$ for the control group, and $6.81 \mathrm{~mm}$ for the vitamin A group $(P=0.390)$. Mean intraoperative pain score using the VAPS was 1.3 in the control group and 1.6 in the vitamin A group $(P=0.901)$.

\section{Postoperative values}

The epithelial healing score at 48 hours was $84.1 \%$ in the control group, and $82.8 \%$ in the vitamin A group $(P=0.706)$. The healing score at day 3 was $97.2 \%$ in the control group, and $95.3 \%$ in the vitamin A group $(P=0.603)$. The mean time to complete healing in the control group was $3.36 \pm 0.6$ days, whereas in the vitamin A group it was $3.42 \pm 0.7$ days $(P=0.854)$. In fact, 26 eyes healed at the third day, two eyes at the second day, and four eyes at the fourth day in the non-vitamin A group; 27 eyes healed at the third day, one eye at the second day, and four eyes at the fourth day in the vitamin A group. No statistically significant difference was found between the two groups $(P=0.838)$. No delayed healing was noted. Mean postoperative pain at the 48-hour visit was $4.35 \pm 1.42$ out of 10 in the control group, and $4.42 \pm 1.37$ out of 10 in the vitamin A group $(P=0.589)$. The mean UCVA at 48 hours, 5 days, 1 week, and 1 month 
Table I Postoperative patient characteristics

\begin{tabular}{lcll}
\hline & $\begin{array}{c}\text { Non-vitamin A } \\
\text { (control) group }\end{array}$ & $\begin{array}{l}\text { Vitamin A } \\
\text { group }\end{array}$ & P-value \\
\hline Spherical error (D) & $-0.35 \pm 0.8 \mathrm{I}$ & $-0.38 \pm 0.69$ & 0.377 \\
3 months & & & \\
Cylinder (D) 3 months & $0.51 \pm 0.54$ & $0.59 \pm 0.6 \mathrm{I}$ & $0.51 \mathrm{I}$ \\
UCVA 48 hours & $0.37 \pm 0.1 \mathrm{I}$ & $0.36 \pm 0.09$ & 0.252 \\
UCVA 7 days & $0.14 \pm 0.012$ & $0.12 \pm 0.014$ & 0.522 \\
UCVA I month & $0.07 \pm 0.012$ & $0.06 \pm 0.008$ & 0.322 \\
UCVA 3 months & $0.023 \pm 0.001 \mathrm{I}$ & $0.04 \pm 0.0012$ & 0.433 \\
BCVA 3 months & $0.026 \pm 0.0014$ & $0.028 \pm 0.0016$ & 0.508 \\
Mean postoperative pain & $4.35 \pm 1.42$ & $4.42 \pm 1.37$ & 0.589 \\
at 48 hours (out of a & & & \\
total score of I0) & & & \\
Mean healing time (days) & $3.36 \pm 0.6$ & $3.42 \pm 0.7$ & 0.854 \\
\hline Abbreviations: D, diopters; UCVA, uncorrected visual acuity; & BVCA, best \\
spectacle-corrected visual acuity. & &
\end{tabular}

are shown in Table 1. The average spherical error at 3 months was $-0.35 \pm 0.81 \mathrm{D}$ in the control group, and $-0.38 \pm 0.69 \mathrm{D}$ in the vitamin A group $(P=0.377)$. The average cylinder at 3 months in the control group was $0.51 \pm 0.54 \mathrm{D}$, whereas in the vitamin A group it was $0.59 \pm 0.61 \mathrm{D}(P=0.511)$.

At 3 months postoperatively, subepithelial haze was graded by the same physician. We found no difference in subepithelial opacity between the two groups $(P=0.960)$. The majority of eyes (93.8\% in both groups) showed a subepithelial opacity below grade 2 (Table 2). No adverse effect secondary to topical vitamin A usage was observed during the study.

\section{Discussion}

Delayed healing, postoperative pain, and corneal haze are the main undesirable complications after excimer laser treatment. ${ }^{13}$ Studies have suggested that keratocytes and epithelial cells are mainly involved in the healing response. In particular, the disappearance of anterior stromal keratocytes in response to excimer laser surgery was an initiating factor, which could then lead to epithelial hyperplasia and eventually to haze formation and regression. ${ }^{14}$ In fact, anterior keratocyte death was mediated by apoptosis with little

Table 2 Postoperative subepithelial opacity 3 months after PRK

\begin{tabular}{lllll}
\hline Grade & $\begin{array}{l}\text { Non-vitamin A } \\
\text { (control) group }\end{array}$ & & $\begin{array}{l}\text { Vitamin A } \\
\text { group }\end{array}$ & P-value \\
\cline { 2 - 2 } & $\mathbf{n}(\%)$ & & n (\%) & \\
\hline 0 & $12(37.5 \%)$ & & $13(40.6 \%)$ & 0.960 \\
0.5 & $13(37.5 \%)$ & & $11(34.4 \%)$ & \\
1 & $5(15.6 \%)$ & $6(18.8 \%)$ & \\
2 & $2(6.2 \%)$ & $2(6.2 \%)$ & \\
3 & 0 & 0 & \\
4 & 0 & 0 & \\
\hline
\end{tabular}

Abbreviations: PRK, photorefractive keratectomy; n, number. accompanying inflammation, and the generation of free radicals was the main cause of apoptosis..$^{15,16}$ The extent of tissue damage, therefore, may reflect the balance between the oxidative damage and the local antioxidation defense system. There is considerable experimental evidence from animal studies to support the use of pharmacological agents that are specifically directed against the oxygen radicals formed during excimer photoablation or that could influence corneal epithelial proliferation and differentiation. ${ }^{17,18}$

On the other hand, although vitamin A exerts a moderate antioxidant activity, it plays an essential part in epithelial growth and limbal stem cell differentiation, promoting corneal wound healing. ${ }^{3}$ Studies suggest that vitamin A may modulate the expression of thrombospondin- 1 in the corneas to accelerate the re-epithelialization of wounded corneas. ${ }^{19}$ On this basis, we attempted to verify the applicability of the data from animal models to the human cornea; we therefore performed the present study to determine whether the use of topical vitamin A could improve corneal re-epithelialization and reduce the postoperative pain and the incidence of haze after PRK.

In the present study, we noted that topical vitamin A at $250 \mathrm{UI} / \mathrm{g}$ neither enhanced corneal healing nor decreased haze formation or pain compared to control (untreated) eyes. In fact, several factors may explain these findings: the vitamin A molecule, the vehicle used, the molecule associated with the active ingredient and the molecule dosage. First, vitamin A is a group of nutritionally unsaturated hydrocarbons that includes retinol, retinal, retinoic acid, and several provitamin A carotenoids. Retinol, the form of vitamin A absorbed when eating animal food sources, is a yellow, fat-soluble substance. Because the pure alcohol form is unstable, it is commercially produced and administered as esters such as retinyl palmitate, or as an alcohol with a petrolatum-based vehicle. In a study conducted by Johansen et al, ${ }^{20}$ all-trans retinoic acid (atRA) was used to investigate its possible healing effect on epithelial corneal abrasions in 13 ocularly healthy Copenhagen White rabbits. This study concluded that atRA $0.05 \%$ applied twice daily increased the healing rate compared to the Vaseline vehicle. This result was not found in our study, which used the retinol palmitate form of vitamin A. In another study conducted by Ubels et al, ${ }^{7}$ an all-trans retinoic acid (Vesanoid [Tretinoin], Roche) formulation in a petrolatum-based ophthalmic ointment vehicle was developed. ${ }^{7}$ This formulation was effective at retinoic acid concentrations of $0.01 \%-0.1 \%$ in reversing corneal keratinization in xerophthalmic rabbits. At $0.1 \%$, it also stimulated the healing rate of corneal epithelial wounds in rabbits and monkeys. ${ }^{20-23}$ 
Second, the specific vitamin A vehicle may play a major role in promoting the delivery of molecules. In a study done by Hattori et al, ${ }^{24}$ inorganically-coated atRA nanoparticles, which are nano-sized egg-like particles of atRA (NANOEGG ${ }^{\circledR}$ atRA; Research Laboratories, Inc, Kawasaki, Japan), were developed. ${ }^{24}$ The purpose of that study was to determine the effects of NANOEGG ${ }^{\mathbb{R}}$-atRA on corneal wound healing on rabbit eyes. Exposure to NANOEGG ${ }^{\circledR}$-atRA decreased the injured area 24 hours after epithelium ablation and was found to promote healing significantly in vitro. In that study, the molecule used and the vehicle were different from those in the present study. Also, the context was different, because results were obtained in vitro and not in vivo, which demands caution for deriving conclusions for human corneal healing.

Third, molecules associated with the active ingredient may affect the healing process. In a randomized controlled experimental study done on rabbit eyes, vitamin A palmitate (VApal) and basic fibroblast growth factor (bFGF) were found to promote repair of mechanical corneal epithelial defects and development of intracellular conjunction. ${ }^{25}$ The effect was more significant when VApal was combined with bFGF. This study used the same molecule used in our study (retinol palmitate), but in conjunction to bFGF. Although this study yielded good results on epithelial healing, concerns should be addressed regarding the context (rabbit eyes) and the experimental model.

Fourth, molecular dosage should also be addressed. A study done by Odaka et $\mathrm{al}^{26}$ suggests that VApal (retinol palmitate) is effective for the improvement of keratoconjunctival epithelial damage in rabbit eyes. The fluorescein staining and rose bengal scores showed a significant decrease in the retinol palmitate group compared with the score in the vehicle group at 7 days $(P<0.05)$ in the $1,000 \mathrm{IU} / \mathrm{mL}$ VApal group and at both 3 days $(P<0.05)$ and 7 days $(P<0.01)$ in the $1,500 \mathrm{IU} / \mathrm{mL}$ VApal group. This study used a higher dosage of retinol palmitate compared with that used in our study ( $250 \mathrm{IU} / \mathrm{g}$, which is equivalent to $312 \mathrm{IU} / \mathrm{mL}$ ) and a different delivery system (eye drops versus petrolatum-based ophthalmic ointment). On the other hand, the study context - using alkaline injury and rabbit eyes - renders the comparison of results with those found in the present study more difficult.

Finally, the route of administration may affect molecule delivery to corneal epithelial cells. Ubels et $\mathrm{al}^{27}$ discovered for the first time that vitamin A is present in the tears as retinol, which corresponds to the normal route of delivery of vitamin A to the cornea in humans and rabbits. This study established the rationale for treatment of corneal disease with topical vitamin A, which endorses the use of the topical route as a preferred one. In fact, we found no significant effect with the topical form at a specific low dosage. On the other hand, the only published clinical trial of oral high-dose vitamin A and vitamin $\mathrm{E}$ supplementation after PRK showed a faster epithelial healing. ${ }^{10}$ This difference could be explained by the different dosage of retinol palmitate used $(25,000 \mathrm{IU}$ per oral supplementation versus $250 \mathrm{IU} / \mathrm{g}$ ), which makes comparison between the two groups difficult. This could be elucidated by measuring retinol concentration in tears after oral administration.

In conclusion, this study showed no effect of retinol palmitate $250 \mathrm{IU} / \mathrm{g}$ with petrolatum-based ophthalmic ointment vehicle on epithelial healing, pain, and haze after conventional PRK. More studies that address the efficacies of different molecular forms of vitamin A, dosage, vehicle, as well as other potentially associated molecules that could enhance vitamin A action, are necessary. The major limitation of this study remains the absence of a sham group (group which receives similar ointment but with no vitamin A) and a third group with only vitamin A with no other treatment (this could only ethically be done in animal studies). Also, other scenarios (for example, chemical burns, traumatism, and infectious keratitis) should be considered in future studies on human eyes to evaluate the possible potential effect of vitamin A on corneal epithelial healing.

\section{Disclosure}

The authors report no conflicts of interest in this work. The study received no external funding. None of the authors has any proprietary, commercial, or financial interest in any of the products mentioned.

\section{References}

1. Ghadhfan F, Al-Rajhi A, Wagoner MD. Laser in situ keratomileusis versus surface ablation: visual outcomes and complications. J Cataract Refract Surg. 2007;33(12):2041-2048.

2. Lee HK, Lee KS, Kim JK, Kim HC, Seo KR, Kim EK. Epithelial healing and clinical outcomes in excimer laser photorefractive surgery following three epithelial removal techniques: mechanical, alcohol, and excimer laser. Am J Ophthalmol. 2005;139(1):56-63.

3. Kruse FE, Tseng SC. Retinoic acid regulates clonal growth and differentiation of cultured limbal and peripheral corneal epithelium. Invest Ophthalmol Vis Sci. 1994;35(5):2405-2420.

4. Hatchell DL, Sommer A. Detection of ocular surface abnormalities in experimental vitamin A deficiency. Arch Ophthalmol. 1984;102(9): 1389-1393.

5. Macsai MS, Agarwal S, Gamponia E. Bilateral corneal ulcers in primary vitamin A deficiency. Cornea. 1998;17(2):227-229.

6. Sommer A, Davidson FR; Annecy Accords. Assessment and control of vitamin A deficiency: the Annecy Accords. J Nutr. 2002;132(Suppl 9): 2845S-2850S.

7. Ubels JL, Edelhauser HF, Foley KM, Liao JC, Gressel P. The efficacy of retinoic acid ointment for treatment of xerophthalmia and corneal epithelial wounds. Curr Eye Res. 1985;4(10):1049-1057. 
8. Herbort CP, Zografos L, Zwingli M, Schoeneich M. Topical retinoic acid in dysplastic and metaplastic keratinization of corneoconjunctival epithelium. Graefes Arch Clin Exp Ophthalmool. 1988;226(1): 22-26.

9. Yoon KC, Im SK, Park YG, Jung YD, Yang SY, Choi J. Application of umbilical cord serum eyedrops for the treatment of dry eye syndrome. Cornea. 2006;25(3):268-272.

10. Vetrugno M, Maino A, Cardia G, Quaranta GM, Cardia L. A randomised, double masked, clinical trial of high dose vitamin A and vitamin E supplementation after photorefractive keratectomy. $\mathrm{Br} J$ Ophthalmol. 2001;85(5):537-539.

11. Kim EJ, Buschmann MT. Reliability and validity of the Faces Pain Scale with older adults. Int J Nurs Stud. 2006;43(4):447-456.

12. Fantes FE, Hanna KD, Waring GO 3rd, Pouliquen Y, Thompson KP, Savoldelli M. Wound healing after excimer laser keratomileusis (photorefractive keratectomy) in monkeys. Arch Ophthalmol. 1990; 108(5):665-675.

13. Epstein D, Fagerholm P, Hamberg-Nyström H, Tengroth B. Twentyfour-month follow-up of excimer laser photorefractive keratectomy for myopia. Refractive and visual acuity results. Ophthalmology. 1994;101(9):1558-1563; discussion 1563-1564.

14. O’Brien TP, Li Q, Ashraf MF, Matteson DM, Stark WJ, Chan CC. Inflammatory response in the early stages of wound healing after excimer laser keratectomy. Arch Ophthalmol. 1998;116(11):1470-1474.

15. Wilson SE, He YG, Weng J, et al. Epithelial injury induces keratocyte apoptosis: hypothesized role for the interleukin-1 system in the modulation of corneal tissue organization and wound healing. Exp Eye Res. 1996;62(4):325-327.

16. Shimmura S, Masumizu T, Nakai Y, et al. Excimer laser-induced hydroxyl radical formation and keratocyte death in vitro. Invest Ophthalmol Vis Sci. 1999;40(6):1245-1249.

17. Brancato R, Schiavone N, Siano S, et al. Prevention of corneal keratocyte apoptosis after argon fluoride excimer laser irradiation with the free radical scavenger ubiquinone Q10. Eur J Ophthalmol. 2000;10(1): $32-38$.
18. Wilson SE, Li Q, Weng J, et al. The Fas-Fas ligand system and other modulators of apoptosis in the cornea. Invest Ophthalmol Vis Sci. 1996;37(8):1582-1592.

19. Uno K, Kuroki M, Hayashi H, Uchida H, Kuroki M, Oshima K. Impairment of thrombospondin-1 expression during epithelial wound healing in corneas of vitamin A-deficient mice. Histol Histopathol. 2005;20(2):493-499.

20. Johansen S, Heegaard S, Prause JU, Rask-Pedersen E. The healing effect of all-trans retinoic acid on epithelial corneal abrasions in rabbits. Acta Ophthalmol Scand. 1998;76(4):401-404.

21. Smolin G, Okumoto M. Vitamin A acid and corneal epithelial wound healing. Ann Ophthalmol. 1981;13(5):563-566.

22. Smolin G, Okumoto M, Friedlaender M. Tretinoin and corneal epithelial wound healing. Arch Ophthalmol. 1979;97(3):545-546.

23. Ubels JL, Edelhauser HF, Austin KH. Healing of experimental corneal wounds treated with topically applied retinoids. Am J Ophthalmol. 1983;95(3):353-358.

24. Hattori M, Shimizu K, Katsumura K, et al. Effects of all-trans retinoic acid nanoparticles on corneal epithelial wound healing. Graefes Arch Clin Exp Ophthalmol. 2012;250(4):557-563.

25. Qiu X, Gong L, Chen M. [Research on effects of vitamin A palmitate on repair of mechanical corneal epithelial defects and conjunctival goblet cells in rabbits.] Zhonghua Yan Ke Za Zhi. 2010;46(2): 151-160. Chinese.

26. Odaka A, Toshida H, Ohta T, et al. Efficacy of retinol palmitate eye drops for dry eye in rabbits with lacrimal gland resection. Clin Ophthalmol. 2012;6:1585-1593.

27. Ubels JL, MacRae SM. Vitamin A is present as retinol in the tears of humans and rabbits. Curr Eye Res. 1984;3(6):815-822.
Clinical Ophthalmology

\section{Publish your work in this journal}

Clinical Ophthalmology is an international, peer-reviewed journal covering all subspecialties within ophthalmology. Key topics include: Optometry; Visual science; Pharmacology and drug therapy in eye diseases; Basic Sciences; Primary and Secondary eye care; Patient Safety and Quality of Care Improvements. This journal is indexed on

\section{Dovepress}

PubMed Central and CAS, and is the official journal of The Society of Clinical Ophthalmology (SCO). The manuscript management system is completely online and includes a very quick and fair peer-review system, which is all easy to use. Visit http://www.dovepress.com/ testimonials.php to read real quotes from published authors. 